\title{
The geomagnetic cutoff rigidities at high latitudes for different solar wind and geomagnetic conditions
}

\author{
W. Chu ${ }^{1,2}$ and G. Qin ${ }^{1}$ \\ ${ }^{1}$ State Key Laboratory of Space Weather, National Space Science Center, \\ Chinese Academy of Sciences, Beijing 100190, China \\ ${ }^{2}$ College of Earth Sciences, University of Chinese Academy of Sciences, Beijing 100049, China \\ Correspondence to: G. Qin (gqin@ spaceweather.ac.cn)
}

Received: 18 May 2015 - Revised: 25 November 2015 - Accepted: 30 November 2015 - Published: 18 January 2016

\begin{abstract}
Studying the access of the cosmic rays (CRs) into the magnetosphere is important to understand the coupling between the magnetosphere and the solar wind. In this paper we numerically studied CRs' magnetospheric access with vertical geomagnetic cutoff rigidities using the method proposed by Smart and Shea (1999). By the study of CRs' vertical geomagnetic cutoff rigidities at high latitudes we obtain the CRs' window (CRW) whose boundary is determined when the vertical geomagnetic cutoff rigidities drop to a value lower than a threshold value. Furthermore, we studied the area of CRWs and found out they are sensitive to different parameters, such as the $z$ component of interplanetary magnetic field (IMF), the solar wind dynamic pressure, $\mathrm{AE}$ index, and Dst index. It was found that both the AE index and Dst index have a strong correlation with the area of CRWs during strong geomagnetic storms. However, during the medium storms, only AE index has a strong correlation with the area of CRWs, while Dst index has a much weaker correlation with the area of CRWs. This result on the CRW can be used for forecasting the variation of the cosmic rays during the geomagnetic storms.
\end{abstract}

Keywords. Magnetospheric physics (energetic particles precipitating)

\section{Introduction}

The ionosphere and atmosphere of the Earth at high and middle latitudes can be affected by the energetic particles which enter the magnetosphere at low altitudes (Nesse Tyss $\varnothing$ y et al., 2013; Galand, 2001). The knowledge of the variation of the energetic particles in the Earth's magnetosphere is important for studying the coupling between the solar wind and the Earth.

The access of energetic cosmic rays into the magnetosphere is controlled by the magnetic field. Störmer (1955) first theoretically described the behavior by assuming the magnetic field is a dipole field. In a pure dipole magnetic field, particles are shielded by a potential barrier from an inner forbidden region whose boundary is defined by the following equation as (Störmer, 1955)

$r=\sqrt{\frac{M q}{m v c}} \frac{\cos ^{2} \lambda}{1+\sqrt{1+\cos ^{3} \lambda}}$,

where $M$ is the dipole moment, $\lambda$ is the geomagnetic latitude, $r$ is the radial distance from the center of the dipole the units of $r$ is the radial distance from the center of the dipole, the units of $r$ are $R_{\mathrm{E}}, c$ is the speed of light, $q$ is the charge of particles, and $v$ is the particle speed. In order to predict the energetic particles transport to a specific location through the magnetosphere, the concept geomagnetic rigidity is used. The geomagnetic rigidity is defined as $R=m v c / q$, where $m$, $v$, and $q$ are the mass, speed, and charge of particle, and $c$ is the light speed.

For each location on the Earth there is a rigidity. In the case that the particles' rigidity is less than the rigidity of a location on the Earth, they cannot reach that location - this rigidity is called geomagnetic cutoff rigidity. From Eq. (1), the cutoff rigidity at any point in space can be expressed as

$R(\mathrm{GV})=\frac{C}{r^{2}} \frac{\cos ^{4} \lambda}{\left(1+\sqrt{1+\cos ^{3} \lambda}\right)^{2}}$,

where constant $C=60$ is derived by the dipole model, and $\mathrm{GV}$ is the unit of the rigidity. It is noted that there are some 
W. Chu and G. Qin: Geomagnetic vertical cutoff rigidity

drawbacks of this model, especially at the high latitudes, because the centered-dipole field is only a rough approximation of the true geomagnetic field, and the influence of the solar wind is not considered. However, precise knowledge of energetic particles in the magnetosphere becomes more and more important for the security of spacecraft and astronauts.

Recently, Smart and Shea did a series of studies (Smart, 1999; Smart et al., 2000; Shea and Smart, 2001; Smart and Shea, 2003a, 2005) to determine the geomagnetic cutoff rigidities from charged particles trajectories by numerically solving Newton's equation of motion. This method is very accurate for calculating cosmic ray cutoff rigidities as long as the magnetospheric field is accurate enough and large-scale computer power is available.

The polar region is very active where energetic particles can access the magnetosphere easily, so it is very interesting to study cosmic ray geomagnetic cutoff rigidities in this region.

Dungey (1962) suggested that the $z$ component (the northward component) of the interplanetary magnetic field (IMF), $B_{z}$ plays an important role in coupling between the solar wind and the magnetosphere. (Kanekal et al., 1998) analyzed the high-latitude energetic particle cutoff rigidity using the data obtained from the SAMPEX satellite and suggested that the parameters of the $B_{z}$ component of IMF and the solar wind speed should be considered. Furthermore, Shimazu et al. (2006) found that the cutoff latitude of $1 \mathrm{MeV}$ protons becomes lower when the $B_{z}$ turns from north to south, while $10 \mathrm{MeV}$ protons do not have such an effect. Tyssøy and Stadsnes (2014) used the measurements from the Medium Energy Proton and Electron Detector on all available Polar Orbit Environment Satellites (POES) to study the variation of the cutoff latitudes and provided a simple applicable parameterization as a function of the Dst index which represents the disturbed magnetic field at the dipole equator on the Earth's surface, $B_{z}$, and solar wind pressure $P_{\text {dyn }}$. However, Kress et al. (2004) found that the smallest cutoff rigidity did not happen at the time when Dst reached the smallest value but $>10 \mathrm{~h}$ prior to the minimum Dst. In addition, Leske et al. (2001) and Kress et al. (2010) found that in the main phase of a geomagnetic storm there is not an obvious correlation between variations of the Dst index and that of the cutoff rigidity.

It is important to study the relationships between the cutoff rigidity and the solar wind, and also between cutoff rigidity and geomagnetic conditions during the geomagnetic storms directly. In this paper, we use the geomagnetic cutoff rigidity to define a cosmic ray window (CRW) at higher latitudes. With the variations of CRW areas we quantitatively study the access of the cosmic rays into the magnetosphere at high latitudes during different interplanetary and geomagnetic conditions. In Sect. 2 the method used is introduced. In Sect. 3 the main results are shown. In Sect. 4 we show the conclusions and discussions of the paper.

\section{Models and methods}

The equation of charged particle's motion in the magnetosphere can be written as

$\frac{\mathrm{d} p}{\mathrm{~d} t}=q v \times \boldsymbol{B}$,

where $p$ is the relativistic momentum of the particle, $v$ is the velocity, $q$ is the charge, and $\boldsymbol{B}$ is the magnetic field. Here the electric field is ignored.

Following Smart et al. (2000) we calculate charged particle trajectories time-backwards with some rigidity at some position on Earth. If a particle can reach the boundary of magnetosphere before its total trajectory path length exceeds $1000 R_{\mathrm{E}}$, the rigidity is considered an allowed rigidity (Kress et al., 2004). However, if the particle intersects the solid Earth or the particle is still moving in the magnetosphere after its total trajectory path length exceeds $1000 R_{\mathrm{E}}$, the rigidity is considered as a forbidden one.

The lowest rigidity above which all rigidities are allowed is denoted as the upper cutoff rigidity $R_{\max }$, and the highest rigidity below which all rigidities are forbidden is denoted as the lower cutoff rigidity $R_{\min }$. From a rigidity much higher than the possible $R_{\max }$ to a rigidity much lower than the possible $R_{\mathrm{min}}$, we scan the rigidity values at the discrete intervals $\delta R=0.001 \mathrm{GV}$. In this way, we obtain the values of $R_{\max }$ and $R_{\min }$, and the allowed and forbidden rigidities between $R_{\max }$ and $R_{\min }$ denoted as $\delta R_{\text {allowed }}$ and $\delta R_{\text {forbidden }}$, respectively. Thus the effective cutoff value, $R_{\text {eff }}$, is calculated with the formula

$R_{\text {eff }}=R_{\max }-\sum_{R_{\min }}^{R_{\max }} \delta R_{\text {allowed }}$.

In our numerical method, Eq. (3) is integrated with the fourth order Runge-Kutta method. The step size in time is set to be equal to 0.01 of particles' gyro-period throughout the trajectory (Kress et al., 2004). The $x, y, z$ coordinates are applicable when using the magnetospheric coordinates which are right handed, orthogonal, Earth centered coordinate systems (Smart et al., 2000). The coordinates we use here are the GEO (geographic coordinate system) and GSM (geocentric solar-magnetospheric).

We consider particles access the position from the vertical direction. So the initial velocities $v_{x_{i}}\left(x_{i}=x, y, z\right)$ of the particles at the given position $x, y, z$ are

$v_{x_{i}}=v \frac{x_{i}}{\sqrt{x^{2}+y^{2}+z^{2}}}$,

where $v$ is the initial velocity of particles, and the altitude of the position is set to be $450 \mathrm{~km}$ as the initial position according to Smart and Shea (2003b). In addition, the inner boundary is set to be $1.0 R_{\mathrm{E}}$ from the Earth center, and the outer boundary is the magnetopause determined by the subroutine 


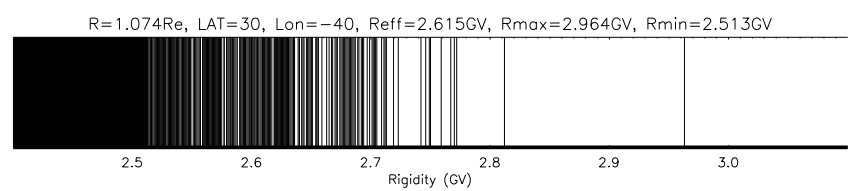

Figure 1. The allowed (white) and forbidden (black) cutoff rigidities in vertical direction at longitude $-40^{\circ}$, north latitude $30^{\circ}$.

T96-MGNP-08 in the GEOPACK-2008 (Tsyganenko, 1995) package.

The magnetospheric field is a combination of the extraterrestrial and internal field. The semi-empirical best-fit magnetospheric model, Tsyganenko 96 (T96) model (Tsyganenko, 1995, 1996; Tsyganenko and Stern, 1996) based on a large number of satellite observations is used as the extraterrestrial background magnetic field. T96 has a realistic shape and size of magnetopause, based on fits to a large number of observed crossings. The T96 magnetic field model is driven by 10 parameters: solar wind pressure $P_{\mathrm{dyn}}$, Dst index, the $B_{y}$ and $B_{z}$ components of the interplanetary magnetic field, and six variables $W_{k}$ which describe individual field sources as timevarying function of geoeffective solar wind parameters.

The internal field model we use here is the international geomagnetic reference field (IGRF) model (http:// www.ngdc.noaa.gov/IAGA/vmod/igrf.html). The model is a standard mathematical description of the Earth's main magnetic field and its secular variation. The IGRF models are standardized for a particular year, reflecting the most accurate measurements available at that time, and indicating a small-scale, slow time variation of the Earth's overall magnetic field. To calculate the internal sources contribution and coordinate transformations we use the package GEOPACK2008 provided by the author of $\mathrm{T} 96$.

All the above computer codes for magnetospheric field and input parameters we use are downloaded from the websites: http://geo.phys.spbu.ru/ tsyganenko/T96. html and http://geo.phys.spbu.ru/ tsyganenko/TS05_data_ and_stuff/. The Dst index is downloaded from http://wdc. kugi.kyoto-u.ac.jp/dstdir.

Figure 1 shows the allowed (white) and forbidden (black) rigidities in vertical direction at longitude $-40^{\circ}$, north latitude $30^{\circ}$. The upper cutoff $R_{\max }=2.964 \mathrm{GV}$, lower cutoff $R_{\min }=2.513 \mathrm{GV}$, and effective cutoff $R_{\text {eff }}=2.615 \mathrm{GV}$. It is shown that cosmic rays are all allowed with vertical rigidities higher than $R_{\max }$, and they are all forbidden with vertical rigidities lower than $R_{\min }$. In addition, they are either allowed or forbidden to access with rigidities between $R_{\max }$ and $R_{\min }$.

As the polar region is very active, here we focus on the vertical rigidities of cosmic rays at high latitudes. Please note that all the following cutoff rigidities are referred to as the effective cutoff rigidities.

Different from the cutoff latitudes we here define a region, cosmic rays window (CRW), where the vertical cutoff rigidi-

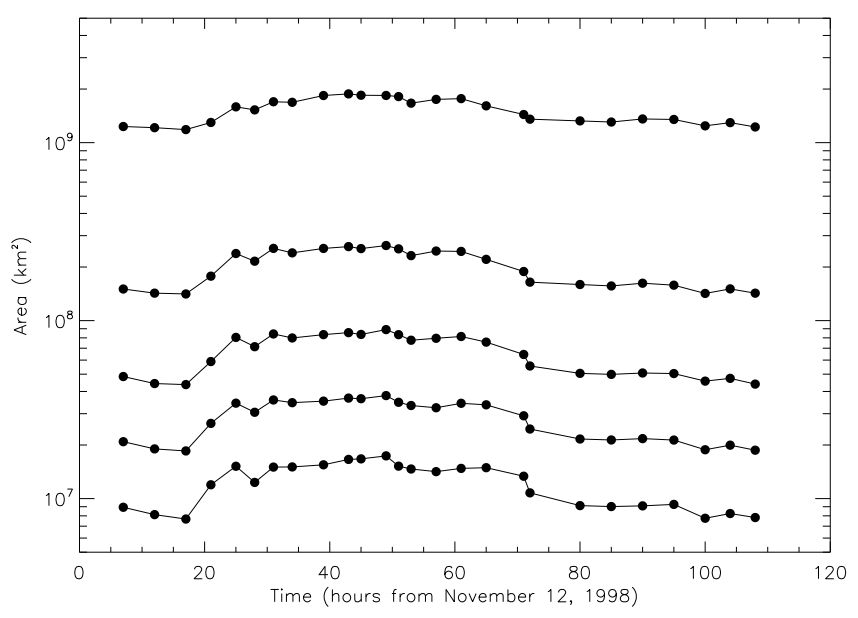

Figure 2. The variations of the area of the CRW for different rigidities. The curves from bottom to top are for values of $M_{t}$ that are equal to $10,20,40,100$ and $500 \mathrm{MV}$, respectively.

ties of energetic particles are less than or equal to a threshold value, $M_{t}$. Figure 2 shows the variations of the area of the CRW for different threshold values, $M_{t}$. The curves from bottom to top, which are for $M_{t}$, equal to 10, 20, 40, 100, and $500 \mathrm{MV}$, respectively. From Fig. 2 we can see that the area of the CRW for different threshold $M_{t}$ has a similar trend of variations. Here, we use a small value, i.e., $20 \mathrm{MV}$, to study the relatively lower energy of cosmic rays. Similar results can be found in both Southern and Northern Hemisphere, and during both quiet and disturbed periods. A simple example is given in Fig. 3, the shaded region indicates the CRW, with the big dot indicating the north magnetic pole.

\section{Results}

It is well believed that solar activity can significantly influence the polar region of the magnetosphere, so the CRW varies with the different parameters of the interplanetary conditions. Therefore, we study CRW with different conditions of solar wind and magnetosphere.

\subsection{Solar wind parameters study of CRW}

We take the period 6 July 2001, 12:00 UT, which is a quiet time with Dst $=6$, to study the area of CRW with varying solar wind parameters, the $z$ component of IMF, $B_{z}$, IMF clock angle, $\theta$, and solar wind dynamic pressure. The IMF clock angle, $\theta$ is defined by $\tan \theta=B_{y} / B_{z}$, where $B_{y}$ is the $y$ component of IMF. The solar wind parameters during the quiet period of 6 July 2001, 12:00 UT are $B_{z}=-0.54 \mathrm{nT}$, $B_{y}=-2.34 \mathrm{nT}$, and $P_{\mathrm{dyn}}=1.65 \mathrm{nPa}$. Below, we study the parameters one by one. In each case, we vary one or two parameters with the rest solar wind parameters kept as the ones of the quiet period, so we get varying CRW corresponding to the varying solar wind parameters. 


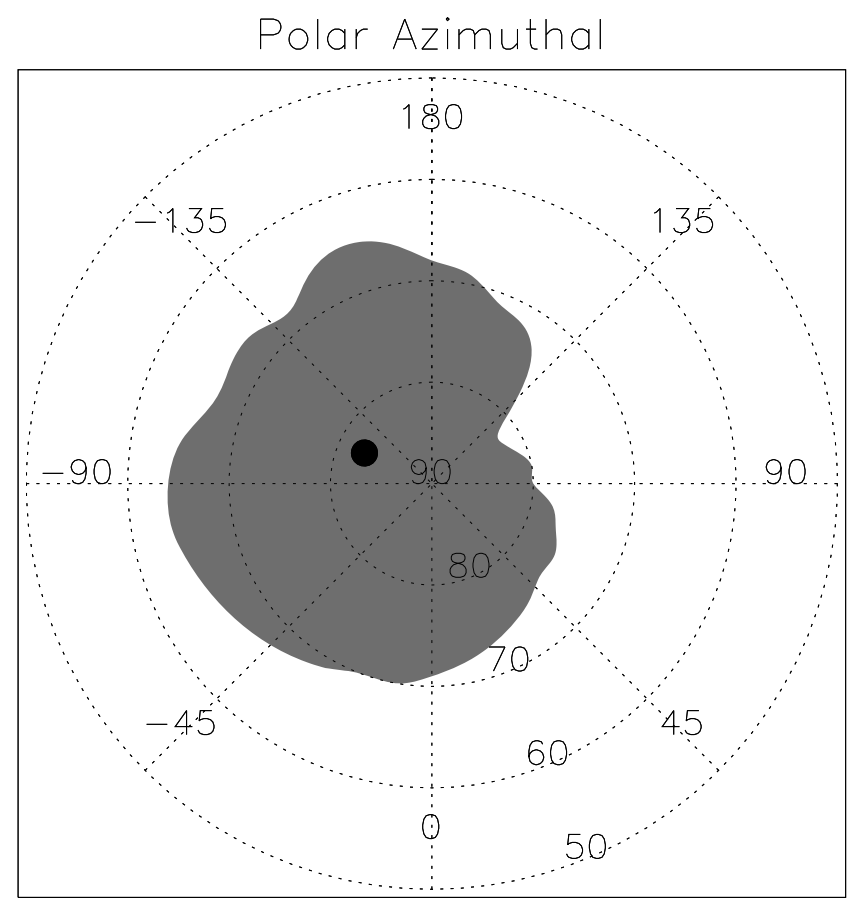

Figure 3. The CRW indicated by the shaded region, with the big dot indicating the north magnetic pole.

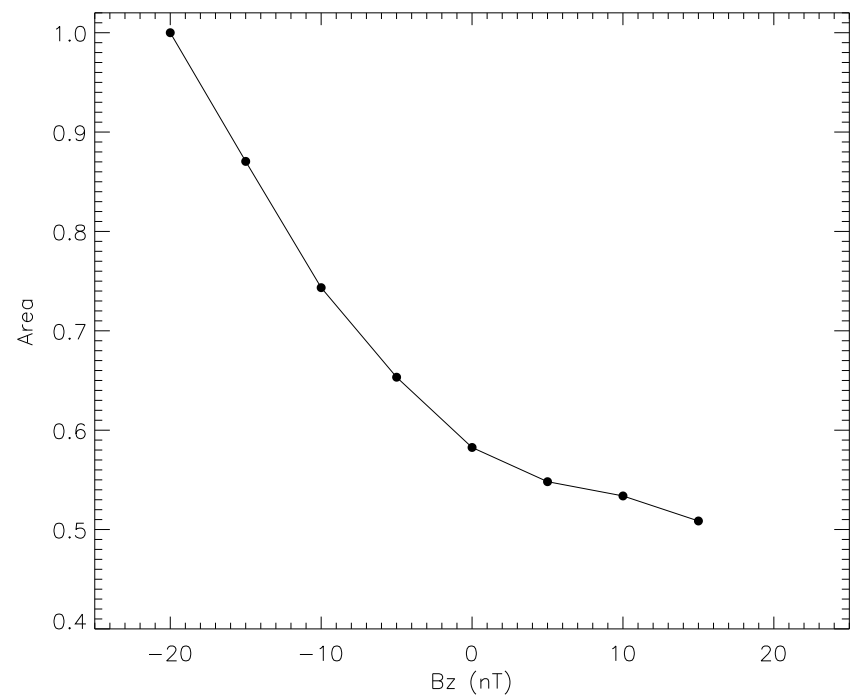

Figure 4. The CRW area as a function of the $z$ component of IMF, all other parameters are the same as that at the quiet time 6 July 2001, 12:00 UT. The $y$ axis has been normalized by the maximum area of CRW during this period.

Figure 4 shows the area of CRW as a function of the $z$ component of IMF, $B_{z}$, where the positive $B_{z}$ indicates the magnetic field pointing to north. Note that in this figure the area of CRW is normalized by the maximum value during the period, and similar normalization is done in other figures. It is shown that the area of CRW decreases with the increas-

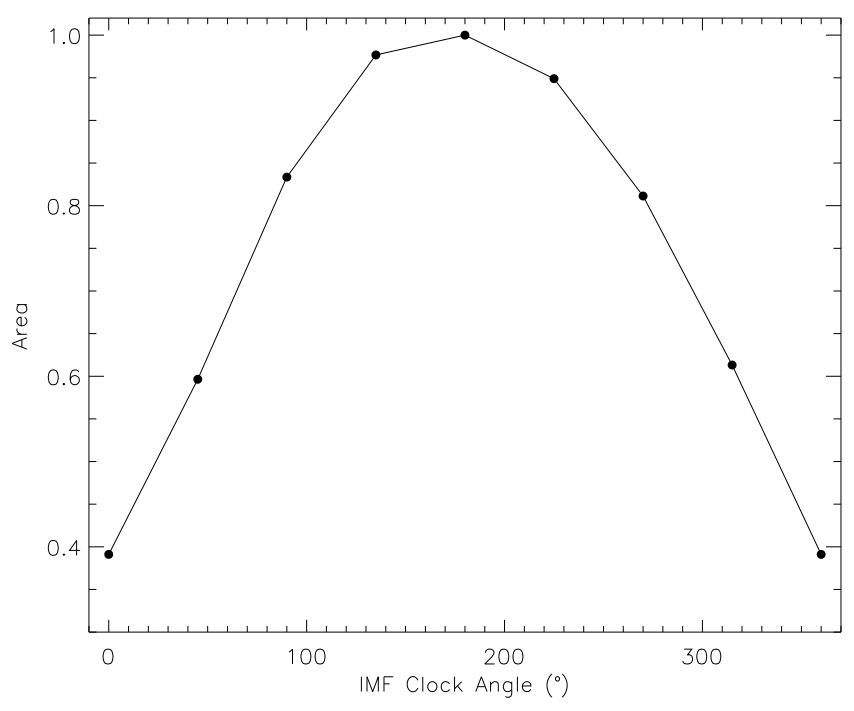

Figure 5. The CRW area as a function of the IMF clock angle, all other parameters are the same as that at the quiet time 6 July 2001, 12:00 UT. The $y$ axis has been normalized.

ing of $B_{z}$. Figure 5 shows the area of CRW as a function of the IMF clock angle $\theta$ which is defined by $\tan \theta=B_{y} / B_{z}$, where $B_{y}$ is the $y$ component of IMF and $B_{y z}=\sqrt{B_{y}^{2}+B_{z}^{2}}$ is a constant (here $20 \mathrm{nT}$ ). It is noted that $B_{z}$ is negative when $90^{\circ} \leq \theta \leq 270^{\circ}$, and $B_{z}$ is positive otherwise. In addition, for any two IMF clock angles, $\theta_{1}$ with $B_{z 1}$ and $B_{y 1}$, and $\theta_{2}$ with $B_{z 2}$ and $B_{y 2}$, if $\theta_{1}+\theta_{2}=360^{\circ}, B_{z 1}=B_{z 2}$ and $B_{y 1}=-B_{y 2}$. From Fig. 5 we can see that the area of CRW is increasing with the decreasing of $B_{z}$, which is consistent with the Fig. 4 . Furthermore, Fig. 5 shows that for conditions with same $B_{z}$ but opposite value of $B_{y}$, the area of CRW is the same. Therefore, from Figs. 4 and 5 we can see that there is a strong anticorrelation between the area of CRW and $B_{z}$, but there is no significant correlation between CRW and $B_{y}$.

Figure 6 shows the relationship between the area of CRW and the solar wind dynamic pressure $P_{\mathrm{dyn}}$ with the solid and dotted lines indicating $B_{z}=5 \mathrm{nT}$ and $B_{z}=-5 \mathrm{nT}$, respectively. From the figure we can see that generally with the same $B_{z}$ the area of CRW increases with the increasing of $P_{\mathrm{dyn}}$. In addition, as shown in previous figures, the area of CRW with negative $B_{z}$ is larger than that with positive one.

Based on the above results we can see that both $B_{z}$ and $P_{\text {dyn }}$ influence the area of CRW, but the determining factor is $B_{z}$.

\subsection{CRW during geomagnetic storms}

Next, we studied the variations of the CRW during three periods of geomagnetic storms. The first geomagnetic storm is a strong one and it occurred on 13 November 1998, with the minimum Dst index -131 at 22:00 UT of that day. Figure 7 shows $B_{z}$ (top panel), $P_{\mathrm{dyn}}$ (second panel), the normalized 


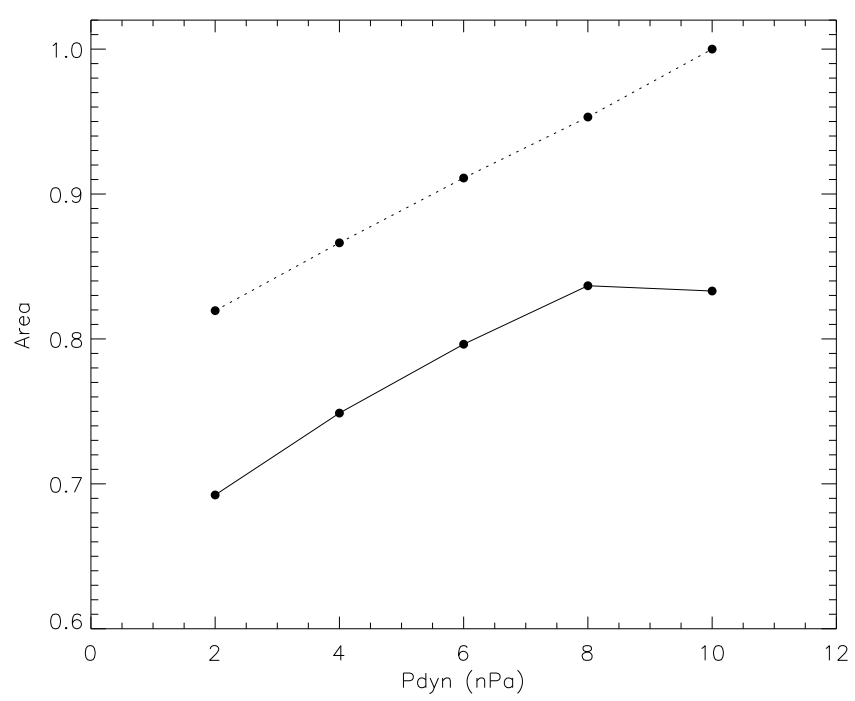

Figure 6. The area of CRW and the solar wind dynamic pressure $\left(P_{\text {dyn }}\right)$ with solid and dotted lines indicating the positive and negative $z$ component of IMF, respectively, all other parameters are the same as that at the quiet time on 6 July 2001, 12:00 UT. The $y$ axis has been normalized.

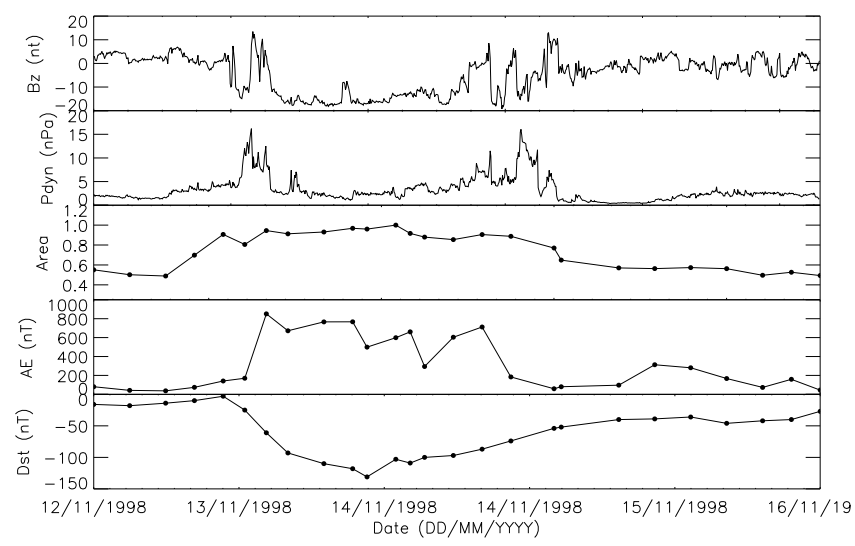

Figure 7. $B_{z}$ (top panel), $P_{\text {dyn }}$ (second panel), the normalized area (third panel), the AE index (fourth panel), and the Dst index (bottom panel) as a function of time during 12-16 November 1998. The $x$ coordinate of black dots in the third panel represents the selected time at which the CRWs are calculated. Note that the $y$ axis of the area panel has been normalized.

area (third panel), the AE index (fourth panel), and the Dst index (bottom panel) for this period. The $x$ coordinate of black dots in the third panel represents the selected time at which the CRWs are calculated.

The second geomagnetic storm, which is also a strong one, occurred on 15 December 2006 with the minimum Dst index -159 at 06:00 UT of that day. The third one is a medium geomagnetic storm and it occurred on 23 January 2012 with the minimum Dst index -69 at 06:00 of that day. Figures 8

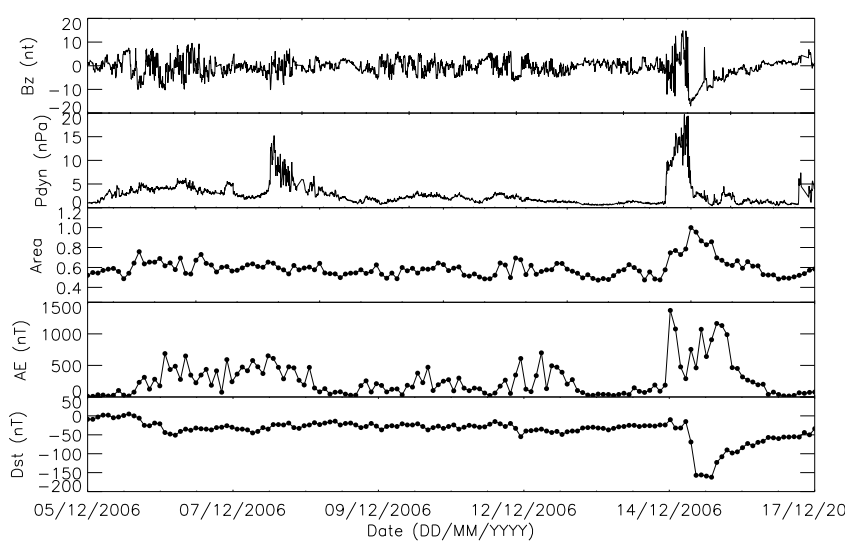

Figure 8. Same as Fig. 7 except during 5-17 December 2006.

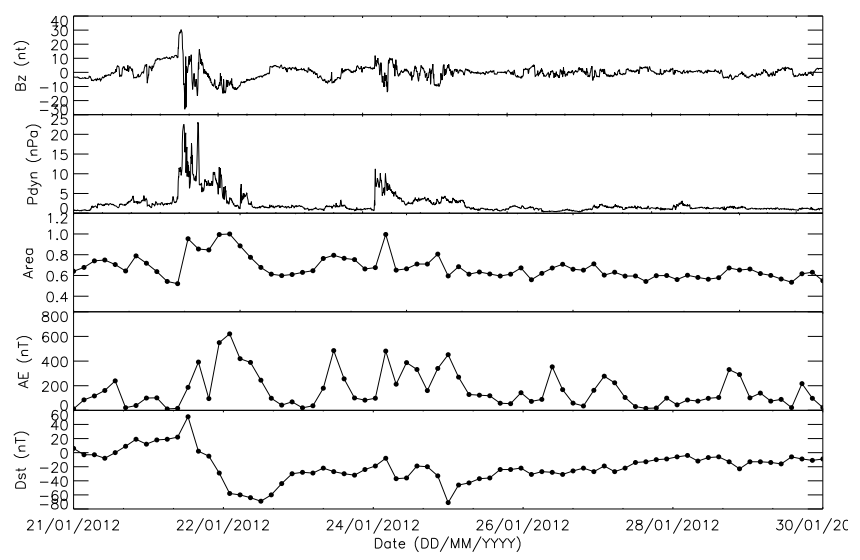

Figure 9. Same as Fig. 7 except during 21-30 January 2012.

and 9 are in the same format as Fig. 7 for the second and third geomagnetic storms, respectively.

Top panels of Figs. 10, 11, and 12 show the variations of the area of the CRWs during the first, second, and third magnetospheric storms, respectively. In the middle and bottom panels of the three figures, we also show AE and Dst indices, respectively. From the figures we can see, during the two strong storms and medium storm, there is a strong correlation between CRW area and the AE index. However, the Dst index is only strongly correlated with the CRW area during the two strong storms.

From Figs. 2 and 7 we can see that the boundary of the CRW will be extended to mid-latitudes during the geomagnetic storm, which is consistent with the result given by Belov et al. (2005).

Then we examined the relationships between the area of the CRW and the solar wind-magnetospheric conditions. In many previous studies (Belov et al., 2005; Pchelkin, 2010; Dmitriev et al., 2010; Kudela et al., 2008) the cutoff rigidity variations during storms have been investigated through the study of the Dst index. In this work, we studied the relationships between cutoff rigidity variations and solar wind- 


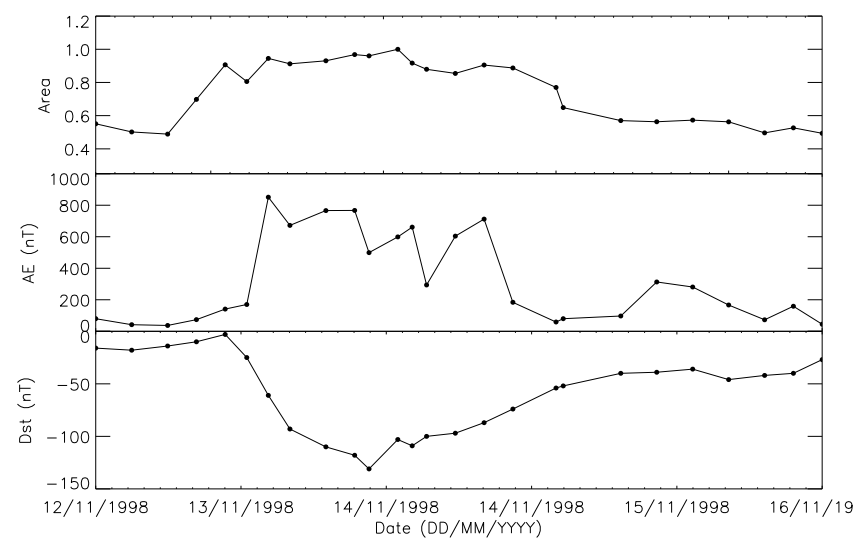

Figure 10. The variations of the area and the $\mathrm{AE}$ and Dst indices during 12-16 November 1998. The black dots represent the selected time to be calculated and the $y$ axis of top panel has been normalized.

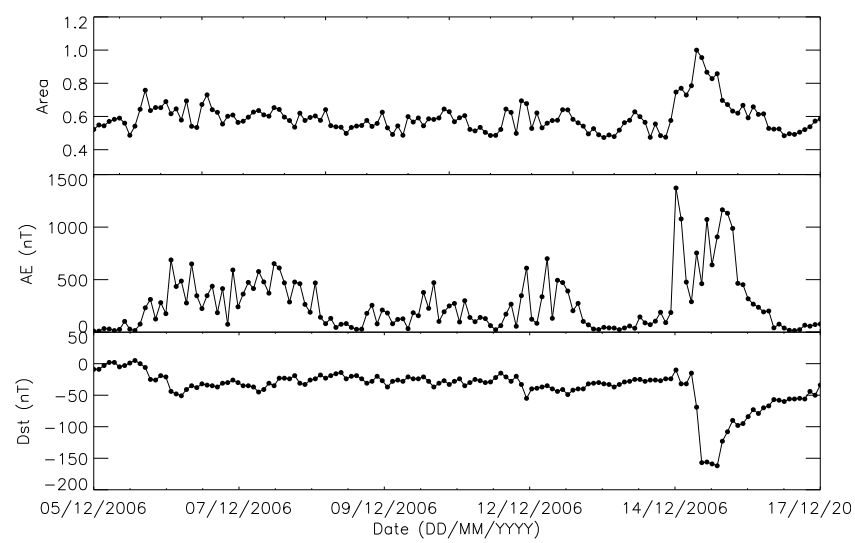

Figure 11. The variations of the area and the $\mathrm{AE}$ and Dst indices during 5-17 December 2006. The black dots represent the selected time to be calculated and the $y$ axis of top panel has been normalized.

magnetospheric conditions such as the $z$ component of the IMF, the solar wind dynamic pressure $\left(P_{\mathrm{dyn}}\right)$, the Dst index and $\mathrm{AE}$ index directly. Such an investigation is quite important since it can provide feedback for space weather applications (Plainaki et al., 2007, 2009; Dmitriev et al., 2010; Tyssøy and Stadsnes, 2014) related to estimation of the atmosphere radiation exposure (Mishev et al., 2011) and to the secondary cosmic ray particles arriving to the ground.

The simulation results show that there exist good relationships between the area of the CRW and the $z$ component of IMF $B_{z}$, and also between the area of the CRW and the solar wind dynamic pressure $\left(P_{\text {dyn }}\right)$. Figure 13 shows the relationship between the area of CRW and the $z$ component of IMF $B_{z}$. It is shown that there is a negative correlation between the area of CRW and $B_{z}$ with $C C=-0.86$ for the linear fitting of area of CRW and $B_{z}$.

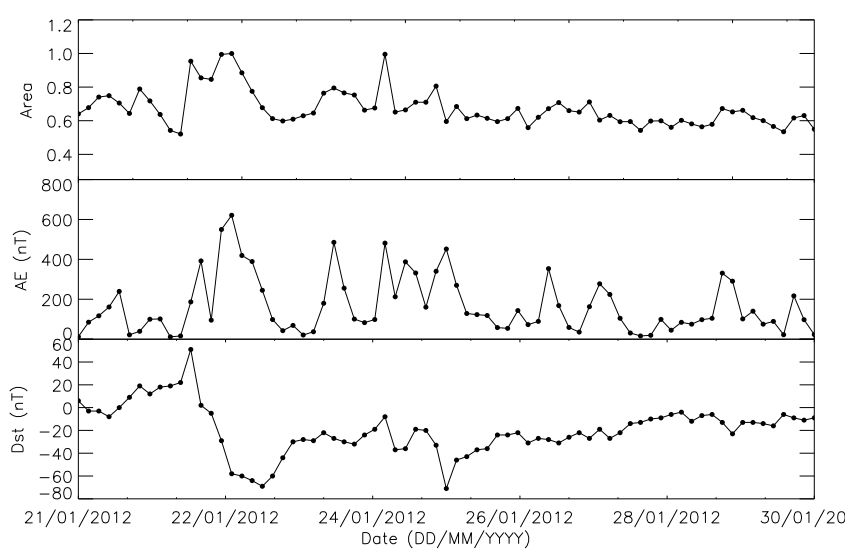

Figure 12. The variations of the area and the AE and Dst indices during 21-30 January 2012. The black dots represent the selected time to be calculated and the $y$ axis of top panel has been normalized.

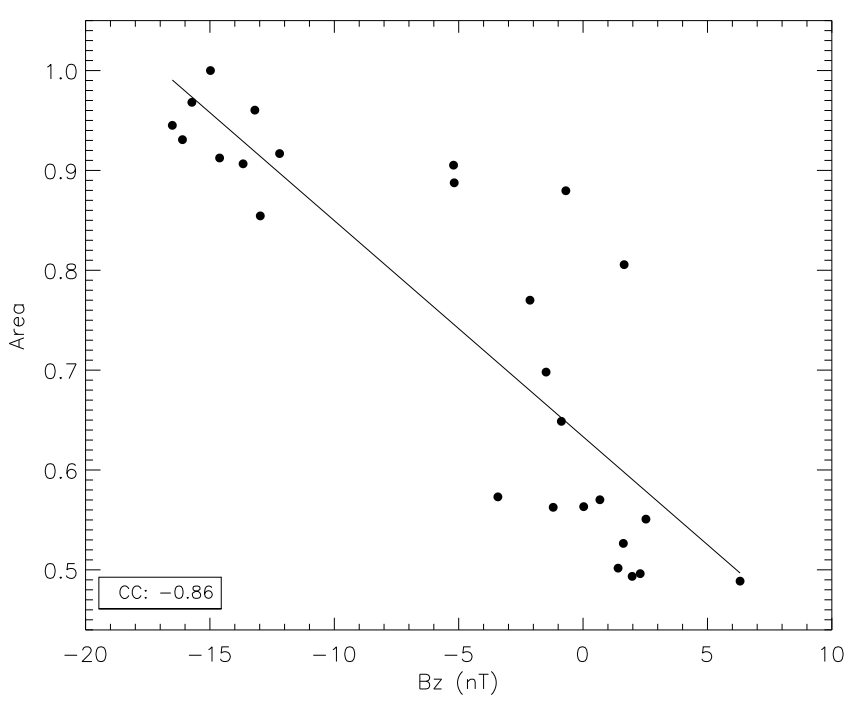

Figure 13. The relationship between the area of CRW and the $z$ component of IMF during different time of geomagnetic storm on 13 November 1998. The $y$ axis has been normalized.

Figure 17 is similar to Fig. 13 except that the $x$ axis is $P_{\text {dyn }}$, from which we can see that approximately there is positive correlation between the area of CRW and the $P_{\mathrm{dyn}}$ parameter.

It is well known that the Dst index is a good index for the magnetosphere storm and it also sometimes has a linear relationship; two of our simulation results also show this phenomenon, but another result shows that it may not be perfect for the study for the CRW sometimes. Figure 16 shows the relationship between the area of CRW and Dst index for another geomagnetic storm on 21 January 2012. We can see that there is no simple relationship between them. We find that the Dst index has a good relationship during the strong geomagnetic storm while there is no such relationship during a medium storm. 


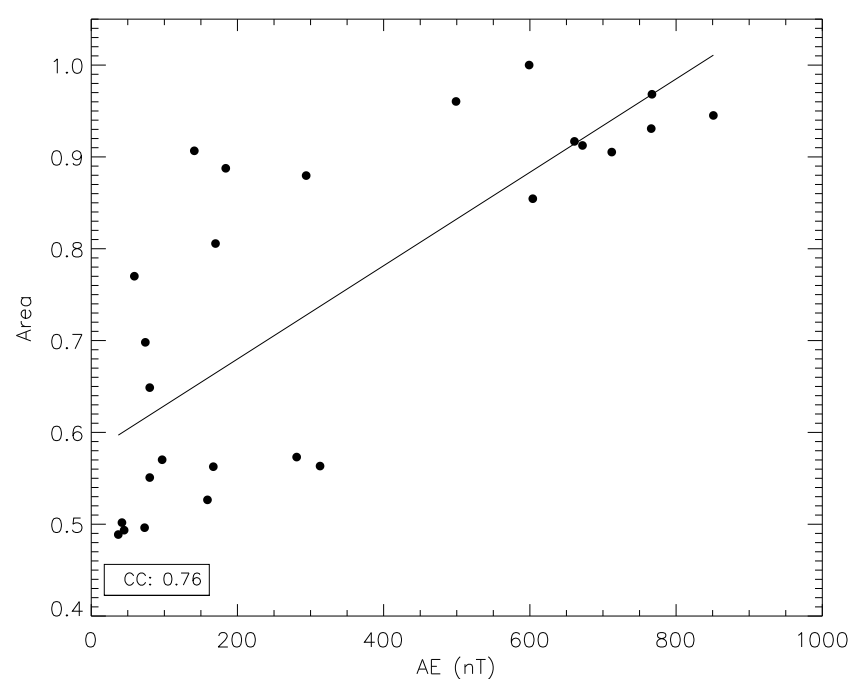

Figure 14. The relationship between the area of CRW and the AE index during different time of geomagnetic storm on 13 November 1998. The $y$ axis has been normalized.

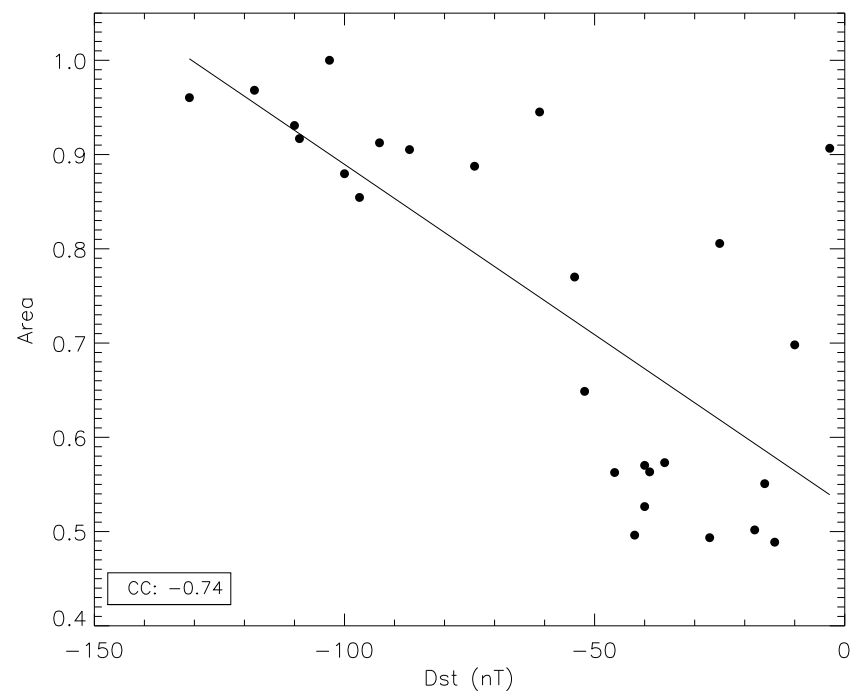

Figure 15. The relationship between the area of CRW and the Dst index during different time of geomagnetic storm on 13 November 1998. The $y$ axis has been normalized.

Instead we find that the auroral electrojet (AE) index is much better than Dst index which is presented in Fig. 14. From this figure we can see that approximately there is a positive correlation between the area of $\mathrm{CRW}$ and the $\mathrm{AE}$ index, with the correlation coefficient $C C=0.76$ for the linear fitting of area of CRW and AE index.

\section{Conclusions and discussions}

In our paper the vertical geomagnetic cutoff rigidities at high latitudes were calculated numerically by solving the trajecto-

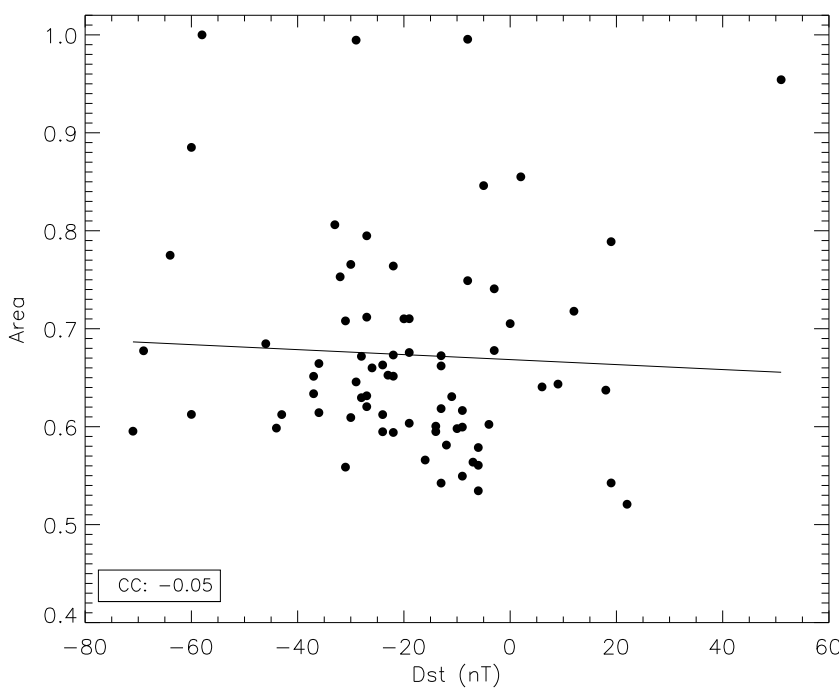

Figure 16. The relationship between the area of CRW and the Dst index during different time of geomagnetic storm on 21 January 2012. The $y$ axis has been normalized. This figure shows that there is no simple linear relationship between the area of CRW and the Dst index.

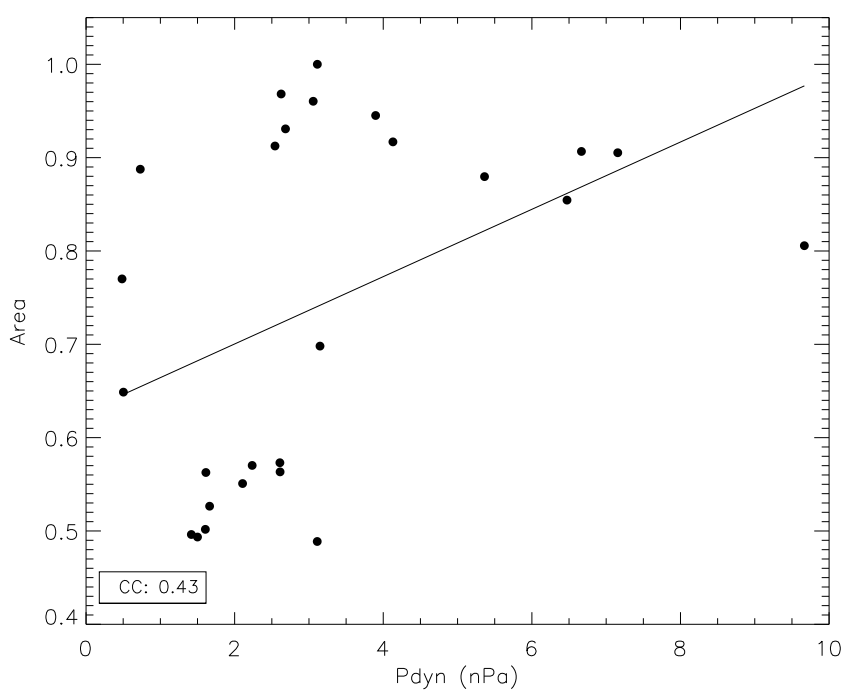

Figure 17. The relationship between the area of CRW and the solar wind dynamic pressure $\left(P_{\mathrm{dyn}}\right)$ during different time of geomagnetic storm on 13 November 1998. The $y$ axis has been normalized.

ries of cosmic rays in the geomagnetic field as a combination of the internal (IGRF 2011) and extraterrestrial model (Tsyganenko geomagnetic field models TS96). A cosmic rays window $(\mathrm{CRW})$ is defined where the vertical rigidities are less than or equal to a threshold value, $M_{t}$. In this paper, we set $M_{t}=20 \mathrm{MV}$.

Our numerical simulations show that the area of the CRW is related to the solar wind parameters, such as the $z$ component of IMF, $B_{z}$, and the solar wind dynamic pressure $\left(P_{\mathrm{dyn}}\right)$, among which $B_{z}$ has the most significant anti-correlation 
with the CRW, but other solar wind parameters (not shown here) do not show as strong correlations with the area of CRW as $B_{z}$ and $P_{\text {dyn }}$ do.

We also studied CRW in different phases of geomagnetic storms in detail. We found that there is generally a positive correlation between the area of CRW and the AE index, and that there is only strong correlation between the area of CRW and the Dst index during strong storms. Therefore, our simulation results suggest that the Dst index is not suitable for forecasting the entry of the energetic particles into the magnetosphere at high latitudes. It is compatible with the results of Kress et al. (2004, 2010); Leske et al. (2001); Merkin and Goodrich (2007). This may be caused by the fact that Dst index represents the disturbed magnetic field at the dipole equator on the Earth's surface; when the geomagnetic storm is not strong enough to affect the ring current the Dst index does not vary much. The particles can, however, enter into the magnetosphere from the polar region. The AE index reflects the activation of the high latitudes so it has better relationships than the Dst index as AE index is a good index for both the strong and medium geomagnetic storms. However the exact reason is still not clear as the magnetosphere is a very complex and dynamic system. This interesting phenomenon needs to be researched in the future.

We also studied the relationship of the neutron monitor count rates and the areas of the CRWs (not shown). However, we found that their correlation is not very strong. The reason might be that our CRW is defined to reflect the entry of lower energetic particles to the magnetosphere rather than that of the relativistic protons. In the future, we would study the entry of much higher energy particles, so that our results could be compared with the neutron monitor count rates (Plainaki et al., 2007; Cordaro et al., 2001; Storini, 2003; Mishev et al., 2011; Vainio et al., 2009; Plainaki et al., 2014, 2015). In addition, the results in this paper may be used to study the effect of the energetic particles on the ionosphere and the atmosphere (Nesse Tyssøy et al., 2013; Galand, 2001) quantitatively. They may also be used to predict the polar cap absorption (PCA) effects (Dmitriev et al., 2010) during the geomagnetic storms. The aircraft radiation dose can also be calculated with a similar method which was adopted by Smart and Shea (2003b); Mertens et al. $(2008,2010)$ and so on.

In future work, we plan to combine the transport of solar energetic particles (SEPs) in interplanetary space (Qin et al., 2004, 2013) as well as the in situ spacecraft SEP measurements at L1 with the access of energetic particles in the magnetosphere, so we may be able to better understand the SEPs influence to the magnetosphere in higher latitudes during space storms.
Acknowledgements. We acknowledge IAGA for the source code of the IGRF model (http://www.ngdc.noaa.gov/IAGA/vmod/igrf. html), Nikolai Tsyganenko for the source code of the T96 model (http://geo.phys.spbu.ru/ tsyganenko/modeling.html), and Kyoto World Data Center for Geomagnetism for Dst indeices (http://wdc. kugi.kyoto-u.ac.jp/index.html). Our work is partly supported by grants NNSFC 41574172, NNSFC 41304135, NNSFC 41374177, and NNSFC 41125016, the CMA grant GYHY201106011, and the Specialized Research Fund for State Key Laboratories of China. The computations were performed by Numerical Forecast Modeling R\&D and VR System of State Key Laboratory of Space Weather and Special HPC work station of Chinese Meridian Project.

The topical editor E. Roussos thanks two anonymous referees for help in evaluating this paper.

\section{References}

Belov, A., Baisultanova, L., Eroshenko, E., Mavromichalaki, H., Yanke, V., Pchelkin, V., Plainaki, C., and Mariatos, G.: Magnetospheric effects in cosmic rays during the unique magnetic storm on November 2003, J. Geophys. Res.-Space, 110, A09S20, doi:10.1029/2005JA011067, 2005.

Cordaro, E., Storini, M., and Olivares, E.: The GLE of July 14, 2000 registered by the Antarctic Laboratory for Cosmic Rays with 1-min time scale, in: International Cosmic Ray Conference, 8, p. 3368, 2001.

Dmitriev, A., Jayachandran, P., and Tsai, L.-C.: Elliptical model of cutoff boundaries for the solar energetic particles measured by POES satellites in December 2006, J. Geophys. Res.-Space, 115, A12244, doi:10.1029/2010JA015380, 2010.

Dungey, J. W.: The interplanetary magnetic field and the auroral zones, Tech. rep., Defense Technical Information Center, Fort Belvoir, VA, 1962

Galand, M.: Introduction to special section: Proton precipitation into the atmosphere, J. Geophys. Res.-Space, 106, 1-6, 2001.

Kanekal, S., Baker, D., Blake, J., Klecker, B., Cummings, J., Mewaldt, R., Mason, G., and Mazur, J.: High-latitude energetic particle boundaries and the polar cap: A statistical study, J. Geophys. Res.-Space, 103, 9367-9372, 1998.

Kress, B., Hudson, M., Perry, K., and Slocum, P.: Dynamic modeling of geomagnetic cutoff for the 23-24 November 2001 solar energetic particle event, Geophys. Res. Lett., 31, L04808, doi:10.1029/2003GL018599, 2004.

Kress, B., Mertens, C., and Wiltberger, M.: Solar energetic particle cutoff variations during the 29-31 October 2003 geomagnetic storm, Space Weather, 8, S05001, doi:10.1029/2009SW000488, 2010.

Kudela, K., Bučík, R., and Bobik, P.: On transmissivity of low energy cosmic rays in disturbed magnetosphere, Adv. Space Res., 42, 1300-1306, 2008.

Leske, R., Mewaldt, R., Stone, E., and Rosenvinge, T.: Observations of geomagnetic cutoff variations during solar energetic particle events and implications for the radiation environment at the Space Station, J. Geophys. Res.-Space, 106, 30011-30022, 2001.

Merkin, V. and Goodrich, C.: Does the polar cap area saturate?, Geophys. Res. Lett., 34, L09107, doi:10.1029/2007GL029357, 2007. 
Mertens, C. J., Wilson, J. W., Blattnig, S. R., Kress, B. T., Norbury, J. W., Wiltberger, M. J., Solomon, S. C., Tobiska, W. K., and Murray, J. J.: Influence of space weather on aircraft ionizing radiation exposure, in: 46th AIAA Aerospace Sciences Meeting and Exhibit, Reno, Nevada, 7-10 January 2008, p. 463, 2008.

Mertens, C. J., Kress, B. T., Wiltberger, M., Blattnig, S. R., Slaba, T. S., Solomon, S. C., and Engel, M.: Geomagnetic influence on aircraft radiation exposure during a solar energetic particle event in October 2003, Space Weather, 8, S03006, doi:10.1029/2009SW000487, 2010.

Mishev, A. L., Velinov, P. I. Y., Mateev, L., and Tassev, Y.: Ionization effect of solar protons in the Earth atmosphere - Case study of the 20 January 2005 SEP event, Adv. Space Res., 48, 12321237, 2011.

Nesse Tyssøy, H., Stadsnes, J., Søraas, F., and Sørbø, M.: Variations in cutoff latitude during the January 2012 solar proton event and implication for the distribution of particle energy deposition, Geophys. Res. Lett., 40, 4149-4153, 2013.

Pchelkin, V.: Effect of magnetospheric substorms on asymptotic directions of arrival of cosmic ray relativistic protons, Geomagn. Aeronomy, 50, 314-319, 2010.

Plainaki, C., Belov, A., Eroshenko, E., Mavromichalaki, H., and Yanke, V.: Modeling ground level enhancements: Event of 20 January 2005, J. Geophys. Res.-Space, 112, A04102, doi:10.1029/2006JA011926, 2007.

Plainaki, C., Mavromichalaki, H., Belov, A., Eroshenko, E., and Yanke, V.: Modeling the solar cosmic ray event of 13 December 2006 using ground level neutron monitor data, Adv. Space Res., 43, 474-479, 2009.

Plainaki, C., Mavromichalaki, H., Laurenza, M., Gerontidou, M., Kanellakopoulos, A., and Storini, M.: The Ground-level Enhancement of 2012 May 17: Derivation of Solar Proton Event Properties through the Application of the NMBANGLE PPOLA Model, Astrophys. J., 785, 160-171, 2014.

Plainaki, C., Laurenza, M., Mavromichalaki, H., Storini, M., Gerontidou, M., Kanellakopoulos, A., Andriopoulou, M., Belov, A., Eroshenko, E., and Yanke, V.: Derivation of relativistic SEP properties through neutron monitor data modeling, in: Journal of Physics: Conference Series, IOP Publishing, 632, 012076, 2015.

Qin, G., Zhang, M., Dwyer, J., and Rassoul, H.: Interplanetary transport mechanisms of solar energetic particles, Astrophys. J., 609, 1076-1081, 2004.

Qin, G., Wang, Y., Zhang, M., and Della, S.: Transport of Solar Energetic Particles Accelerated by ICME Shocks: Reproducing the Reservoir Phenomenon, Astrophys. J., 766, 38-38, 2013.
Shea, M. and Smart, D.: Vertical cutoff rigidities for cosmic ray stations since 1955, in: International Cosmic Ray Conference, Hamburg, Germany, 7-15 August 2001, 10, 4063, 2001.

Shimazu, H., Tanaka, T., Den, M., and Obara, T.: Dependence of the cutoff latitude of solar energetic protons on the southward component of the IMF, Adv. Space Res., 38, 503-506, 2006.

Smart, D.: Changes in calculated vertical cutoff rigidities at the altitude of the international space station as a function of geomagnetic activity, in: International Cosmic Ray Conference, Salt Lake City, Utah, USA, 17-25 August 1999, 7, 337, 1999.

Smart, D. and Shea, M.: The limitations of using vertical cutoff rigidities determined from the IGRF magnetic field models for computing aircraft radiation dose, Adv. Space Res., 32, 95-102, 2003a.

Smart, D. and Shea, M.: The space-developed dynamic vertical cutoff rigidity model and its applicability to aircraft radiation dose, Adv. Space Res., 32, 103-108, 2003 b.

Smart, D. and Shea, M.: A review of geomagnetic cutoff rigidities for Earth-orbiting spacecraft, Adv. Space Res., 36, 2012-2020, 2005.

Smart, D., Shea, M., and Flückiger, E.: Magnetospheric models and trajectory computations, Space Sci. Rev., 93, 305-333, 2000.

Storini, M.: Cosmic-ray Characteristic Parameters for Yangbajing (Tibet) Experiments, in: International Cosmic Ray Conference, Trukuba, Japan, 31 July-7 August 2003, 7, 4283, 2003.

Störmer, C.: The polar aurora, Clarendon Press, Oxford, UK, 1955.

Tsyganenko, N. A.: Modeling the Earth's magnetospheric magnetic field confined within a realistic magnetopause, J. Geophys. Res.Space, 100, 5599-5612, 1995.

Tsyganenko, N. A.: Effects of the solar wind conditions in the global magnetospheric configurations as deduced from databased field models, in: International Conference on Substorms, Versailles, France, 12-17 May 1996, 389, 181, 1996.

Tsyganenko, N. A. and Stern, D. P.: Modeling the global magnetic field of the large-scale Birkeland current systems, J. Geophys. Res.-Space, 101, 27187-27198, 1996.

Tyss $\varnothing y$, H. N. and Stadsnes, J.: Cutoff latitude variation during Solar Proton Events: Causes and Consequences, J. Geophys. Res.Space, 120, 553-563, 2014.

Vainio, R., Desorgher, L., Heynderickx, D., Storini, M., Flückiger, E., Horne, R. B., Kovaltsov, G. A., Kudela, K., Laurenza, M., McKenna-Lawlor, S., Rothkaehl, H., and Usoskin, I. G: Dynamics of the Earth's particle radiation environment, Space Sci. Rev., 147, 187-231, 2009. 